Instituto Internacional de Investigación y Desarrollo Tecnológico Educativo INDTEC, C.A.

DOI: https://doi.org/10.29394/scientific.issn.2542-2987.2016.1.1.13.231-248

OAI-PMH: http://www.indteca.com/ojs/index.php/Revista Scientific/oai

\title{
Gerencia Deportiva para la Masificación Deportiva Planificada y Ejecutada por las Organizaciones Sociales. Críticas a Modelos, Experiencias y Propuesta de Acompañamiento Metodológico
}

\author{
Autora: Lorenza Antonia Reyes de Duran \\ Universidad Nacional Experimental del Yaracuy, UNEY \\ lorenzareyes1@yahoo.com \\ San Felipe, Venezuela
}

\section{Resumen}

La presente propuesta de análisis, interpretación, desmontaje, autocrítica y orientación nace y surge de experiencias de trabajo de masificación deportiva planificadas en y por organizaciones sociales, contrapuestas-no en sentido comparativo- a los modelos convencionales de gerencia deportiva, estatal y empresarial-privada. El aporte que hacen las experiencias de gerencia deportiva desde espacios de poder, bien sea estatal o empresarial son innegables y su impacto es difícil de expresar en números por su valor humanista, el cual es incalculable. No obstante, es urgente recalcar los productos y resultados alcanzados por algunas organizaciones sociales vinculadas a la actividad deportiva; como constituyen los casos referenciales en Higuerón y en el municipio Independencia del estado Yaracuy. Desde un análisis dialéctico de la realidad, tratamos de comprender el complejo sistema que supone la gerencia deportiva de alto nivel y rendimiento, nos introduciremos en las nociones práxicas de la actividad deportiva asociada y aplicada al enfoque del trabajo social y comunitario. Como cierre y apertura de procesos de investigación-acción, haremos una propuesta de acompañamiento a referentes de gerencia de masificación deportiva por y desde las organizaciones sociales. Esta propuesta se construye desde un diseño curricular novedoso, flexible, abierto, integrador y con pertinencia social, comunitaria y organizacional.

Palabras clave: organización deportiva; gerencia deportiva; masificación deportiva; planificación socio-comunitaria; organización social; diseño curricular. 


\title{
Sports Management for Sports Massification Planned and Executed by Social Organizations. Critics to Models, Experiences and Proposal Methodological Accompaniment
}

\begin{abstract}
The proposal analysis, interpretation, disassembly, self-criticism and guidance is born and comes from work experience planned mass sports and social organizations opposed-not in the conventional sense comparativeprivate business models and sport, state and management. The contribution made by the sports management experience from positions of power, either state or business are undeniable and its impact is difficult to express in numbers for its humanistic value, which is incalculable. However, it is urgent to emphasize the products and results achieved by some social organizations related to sport; as are the reference cases in Higuerón and the municipality Independence of Yaracuy state. From a dialectical analysis of reality, we try to understand the complex system that involves high-level sports management and performance, we introduce in the praxical notions of sporting activity and associated approach applied to social and community work. As opening and closing action research processes, we will make a proposal to accompany sports management concerning overcrowding by and from social organizations. This proposal is constructed from an innovative, flexible, open, inclusive and social, community and organizational curriculum relevance.
\end{abstract}

Keywords: sports organization; sports management; sports massification; socio-community planning; social organization; curricular design. 


\section{Introducción}

Toda actividad humana es acción cultural. La cultura está construida por códigos, lenguajes, expresiones, manifestaciones, formas organizativas, lo identitario, la mentalidad, en sí, es dinámica, diversa; pero también es vulnerable y manipulable por otros aspectos que inciden en la sociedad humana y en las relaciones sociales de producción.

Estas relaciones sociales de producción vienen a ser definidas por la estructura configurada por la actividad económica, el sistema social y el sistema político y lo cultural es la superestructura de tales relaciones que definen un modelo socio-político. La educación y la producción cultural sostienen a la superestructura. En tal sentido, el hecho deportivo en su dimensión lúdica natural y no como elemento diseñado por las políticas de Estado, ni como excusa para la responsabilidad social de la empresa privada, es una de las bases de la acción cultural.

En este instrumento para la reflexión y el análisis sobre las relaciones sociales, se realizarán planteamientos técnicos, teóricos, críticos y propuestas para el acompañamiento requerido en la masificación deportiva.

De antesala podemos afirmar que la actividad deportiva- así como por ejemplo la actividad artística- sufren permanentemente una tensión en cuanto a la realidad de ser vulnerables en referencia a su uso como propaganda proselitista del Estado y al mismo tiempo, es secuestrada y convertida en negocio y mercancía por la industria cultural de la televisión al servicio de las grandes corporaciones.

\section{Nociones práxicas}

En este artículo acudiremos a nomenclaturas y categorías que definen las tareas propias de la gestión deportiva y de la actividad física y su vinculación con otros aspectos. 
En tal sentido, proponemos una redefinición de términos por las características de este producto académico:

\subsection{Actividad Física y Deportiva:}

Es un conjunto de acciones direccionadas o no, concebidas, aplicadas y ejecutadas de forma individual, con el fin de ejercitar el cuerpo humano. Esta actividad física es dinámica, pues incorpora técnicas convencionales, alternativas, creativas, holísticas, nutricionales-gastronómicas, religiosasancestrales y está adecuada a las necesidades corporales, mentales y espirituales de quienes se involucran en la actividad física.

La definición de actividad física será sencilla y breve; (Cagigal, 1981: 88): "Conjunto de acciones con objetivos motores". 0 , de una manera más explícita, "conjunto de realizaciones de las personas cuyas intenciones son hacer participar su motricidad". Lo cual quiere decir que cada actividad física y deportiva tiene sus propios tipos de acciones identificadas, provocadas, caracterizadas, orientadas y guiadas según sus propios objetivos motores.

\subsection{Cultura:}

Es entendida como el producto de las relaciones de producción, como un fenómeno que no está desligado del modo de producción de una sociedad. Asimismo, la considera como uno de los medios por los cuales se reproducen las relaciones sociales de producción, que permiten la permanencia en el tiempo de las condiciones de desigualdad entre las clases. Es una de las formas de control hegemónico de las élites estatales, empresariales, transnacionales y de los consorcios industriales.

En el caso de la actividad física y deportiva, la industria del entretenimiento, secuestra toda expresión, inventa formas de patentes, se dan derechos de transmisión de los grupos monopólicos, el atleta es convertido en una mercancía-objeto sometido a las lógicas cambiantes del rendimiento 
físico, de la taquilla, de las marcas comerciales y se crea todo un meta-relato transformando las disciplinas deportivas en fenómenos religiosos que dan soluciones.

\subsection{Cultura Deportiva:}

Es el resultado o producto de todo un complejo sistema de relaciones sociales de producción que se dan en torno al hecho deportivo en sus diversos niveles: popular-espontáneo, direccionado por el Estado o por la industria del entretenimiento.

Existe una tensión entre los diversos enfoques sobre la finalidad de la cultura deportiva en cuanto a los objetivos, políticas, valoración, papel en la transformación de la sociedad y los sujetos, el rol preventivo, la alta competencia y la veracidad de su impacto, el hecho de dejarse absorber 0 ceder ante las lógicas culturales hegemónicas, así como la tarea política del deporte y desmontaje del mito de la neutralidad ideológica y de ser una actividad no-lucrativa.

\subsection{Deporte:}

Actualmente, debido al gran auge de la actividad físico-deportiva, el deporte es analizado desde diferentes puntos de vista, ya sea desde el ámbito cultural como el científico. De este modo el deporte es estudiado por la sociología, la filosofía, la biomecánica, la educación, la historia, etc. Como afirma (García 1990), el deporte abarca las distintas manifestaciones en diversos órdenes de nuestra sociedad, de manera que en los últimos años se habla de la industria deportiva como grupo diferenciado que ofrece actividades de distracción, entretenimiento, espectáculo, educación, pasatiempo y, en general, bienes y servicios relacionados con el ocio y la actividad física, competitiva y recreativa. 
El término deporte, con el que en la actualidad nos referimos a algo tan claro y que evidencia un fenómeno sociocultural y educativo por todos conocidos, es una de las palabras más polémicas, además de ser uno de los términos más fascinante y complejo (Paredes, 2002).

\subsection{Gerencia Deportiva:}

Es un conjunto de acciones y tareas articuladas desde el hecho deportivo en sus diversos y múltiples espacios, niveles, modalidades y formatos en parámetros de calidad gerencial.

La calidad gerencial, (según Bustamante 2008) se evalúa a través de la capacidad para detectar desafíos, oportunidades, y formular objetivos claros y cumplirlos. Se requiere pues, de la supervisión y control constante al conjunto de tareas individuales para asegurar el alcance de esos objetivos. La gerencia desarrollada sobre la base de la transferencia de información y de comunicación oportuna de los planes, programas y proyectos, constituiría la base para hacer una eficiente distribución de poder, no solo porque diversificaría las tareas a desarrollar, sino también porque faculta para la toma de decisiones en tanto que, fundamenta la interacción social, se descubre potencialidades, fomenta el trabajo en equipo y esto coadyuva con los procesos de cambio para la productividad en todos los órdenes, además que permite explorar, experimentar e innovar.

En ese orden de ideas y planteamientos la gerencia deportiva será de calidad no sólo cuando quienes direccionan y operativizan los planes y proyectos cuentan con las condiciones propias de los ambientes estatales y empresariales. Esta tiene desafíos de ser efectiva, eficaz y de calidad en la medida que responde a otras demandas y exigencias y tenga la capacidad de adecuarse a lógicas propias de los territorios y públicos. 


\subsection{Organizaciones Sociales:}

Aproximándonos a la conceptualización de (Pfeffer 1993) y en base a la comprensión de la observación e interpretación de los procesos propios producidos en las comunidades, se pudiera construir y precisar que las organizaciones sociales son expresiones de encuentro de las y los ciudadanos con el objetivo de participar en el análisis de problemas para buscar una toma de decisiones a partir de la planificación de actividades orgánicas, articuladas y también aisladas dependiendo las condiciones, metas y compromiso de los actores involucrados.

En la concreción de Venezuela, existe una gran diversidad de organizaciones sociales de base con múltiple intención, cooperativas, asociaciones y fundaciones desinadas a la promoción artística, educativa, capacitación, transporte, producción, servicios, religiosas, deportivas y recreativas que operan en algunos casos, desde hace más de 100 años.

Desde hace 10 años han surgido otras formas de organización social conocidas como expresiones del Poder Popular, como es caso de los consejos comunales y de las comunas. Estas formas se desarrollan en la lógica del ámbito territorial, las mencionadas anteriormente tienen otro alcance por sus características y naturaleza.

\subsection{Relaciones Sociales de Producción:}

Son aquellas que se establecen entre los propietarios de los medios de producción y los productores directos del proceso de producción determinado. Es decir, los propietarios viven a costa del trabajo de los productores directos.

En el caso del espectáculo deportivo direccionado por la industria del entretenimiento, se puede afirmar que el atleta es quien genera riqueza a los dueños de los medios de producción (televisión, marcas, anunciantes y propietarios de las empresas deportivas) con su trabajo y preparación física. 
El modo de producción convierte al atleta en mercancía, entendiéndola como un conjunto de productos que son valores de uso para otros a través del mercado. Es decir, que un producto, con su 'valor de uso', adquiere al ser vendido un valor de cambio, reflejado en el precio. La acción legal-comercial de firmar a un atleta es una transacción donde la empresa deportiva compra por un tiempo determinado la exclusividad de la fuerza productiva y creativa de este.

\section{Desmontaje del espectáculo deportivo}

El deporte como espectáculo, según Soriano (2009) y (Borrel y Cañadas 2010) se caracteriza por su condición de distracción, fiesta, representación, función y diversión de las personas que asisten a él.

El deporte como consumo y espectáculo de masa, es uno de los mayores espectáculos de nuestro tiempo. Uno de los medios más asequibles y económicos de entretenimiento de la sociedad de masas. No ha alcanzado todavía los límites en los que se detenga su crecimiento. El ocio se ha convertido en "mercancía de consumo", y dentro de este, el deporte juega un papel fundamental. Es beneficioso económicamente, objeto de intereses económicos, comercialmente atractivo y con capacidad para competir en mercados dinámicos.

\section{Crítica a las gerencias deportivas}

$\mathrm{Si}$ bien es cierto que la gerencia deportiva obedece a intereses opuestos 0 distintos cuando contraponemos lo propio a las funciones y características del sector estatal en sus niveles de gobierno frente $o$ al lado de las especificidades del sector privado de la industria del entretenimiento deportivo en sus estrategias de captación, se pueden establecer también puntos de coincidencia en cuanto a formas, métodos y actores. 
5. La masificación deportiva autogestionable en las comunidades, experiencias en comunidades del estado Yaracuy.

\subsection{Descripción sistematización y de las experiencias:}

En estas últimas décadas de la historia geopolítica global, se ha insistido en el uso y difusión posicional de ciertas categorías sociales, políticas, antropológicas, culturales y epistémicas, tales como:

- Poderes creadores del Pueblo

- Democracia protagónica y participativa

- Empoderamiento del pueblo

- Democratización de técnicas

- Intercambio y transferencia de saberes

- Innovaciones científicas

- Patrimonios vivientes y cultores

- Saberes y conocimientos ancestrales

- Igualdad de condiciones, inclusión social y acceso a la información

- Visibilización de sujetos emergentes

- Tejido social

Para muchos, estos términos resultan novedosos; pero si investigamos en nuestros ámbitos locales nos encontraremos con un acumulado histórico de experiencias significativas de organización social en torno a las actividades caracterizadas como parte estratégica del desarrollo social: salud, artes, educación, cultura, deporte, recreación, religiosidad y mejoras en la infraestructura y servicios.

Este acumulado de organización social está expandido por todo el país y nos atrevemos a afirmar responsablemente y desde nuestra subjetividad, que ha servido de plataforma para construir experiencias políticas, sociales y de desarrollo económico. 
En lo concerniente a nuestro tópico denominado la gerencia deportiva, será injusto desconocer sobre el aporte del trabajo de promoción deportiva desarrollado en las comunidades por promotores y dirigentes de las bases, como insumos para la aplicación de las políticas estatales y empresarialesprivadas en materia de competencia deportiva.

Las experiencias de gerencia deportiva autogestionables no gubernamentales ni empresariales, a diferencias de la alta gestión o dirección institucional, no depende de una agenda sujeta a intereses de los gobiernos ni de la rentabilidad de la industria del entretenimiento deportivo.

Antes de seguir profundizando en este aspecto sistematizador, es pertinente acudir a mencionar de forma concisa algunas experiencias ejemplificantes de gerencia deportiva sociocomunitaria en el estado Yaracuy.

Como primera experiencia o referencia, se menciona la de la comunidad de Higuerón, ubicada en la parroquia capital del municipio San Felipe, denominada "Juegos Vacacionales de Higuerón".

Estos juegos se vienen realizando desde agosto del año 1991. Han sido 25 ediciones hasta la fecha. El equipo organizador lo integran una docena de vecinos al que se suman árbitros y colaboradores. Entre las personas que llevan la tarea de gerenciar ese programa, se encuentran los promotores deportivos y sociales Luis Sira, Luis Sira Navarro y Renny Hernández.

Los juegos vacacionales tienen una duración de 2 meses y el cronograma de encuentros se desarrolla desde las 9:00 am hasta las 10:00 $\mathrm{pm}$, ofreciendo a los espectadores competencias de las disciplinas de fútbol sala, volibol y baloncesto, con participación de atletas desde los 8 años de edad hasta categoría libre, masculino y femenino.

\section{Interpretación de las experiencias y resultados}

Para interpretar las experiencias y aproximarnos al enunciado de resultados, acudiremos desde un enfoque cualitativo, a las instrucciones que 
encontramos en las propuestas metodológicas de investigación social como (Acosta 2012), (Pfeffer 1993) y (Soriano 2009); donde se indica hacer un esfuerzo por indagar sobre las percepciones y motivaciones que orientan a las organizaciones sociales, en nuestro caso, a planificar y ejecutar actividades para la masificación deportiva.

Nos hacemos las preguntas generadoras: ¿Qué motiva internamente a los promotores que gestionan estos juegos? ¿Cuál es el fin de los atletas que participan en este tipo de eventos, sabiendo que esta actividad no es vista como una plataforma para ser captados como prospectos o como atletas de alto rendimiento? ¿Cómo se podría dar sustentabilidad a la realización de eventos que promuevan la masificación deportiva?

En la lógica de la gerencia deportiva estatal estas experiencias son apoyadas sólo en épocas que coinciden con procesos electorales y en la visión de la gerencia deportiva empresarial del sector privado, estos juegos aun cuando tienen una cobertura territorial importante, no representan un atractivo para el negocio de la industria del espectáculo deportivo. Los funcionarios que gerencian la actividad física y deportiva en ambos espacios (estatal y privado) práxicamente perciben estas competencias como un gasto de recursos y un desgaste de energías.

Al entrevistar y aplicar una encuesta abierta a un universo de 300 consultados entre promotores deportivos (organizadores, árbitros y entrenadores; así como a deportistas provenientes de los diversos equipos de las comunidades involucradas en las experiencias referenciales citadas en este trabajo podríamos señalar varias actitudes.

A los consultados se les preguntó: ¿Cuáles son los aprendizajes que usted cree, dejan como resultado la organización, planificación y ejecución de actividades de masificación deportiva?. Mencione 3 indicadores.

Los promotores deportivos y sociales y los atletas de las diversas comunidades, que tienen como sustento otras actividades económicas, es 
muy probable vean a la actividad física y deportiva como una genuina posibilidad para:

- $(89,99 \%)$ El intercambio fraterno y amistoso.

- $(87,99 \%)$ El combate a las drogas y la violencia como tareas preventivas.

- $(63,66 \%)$ La construcción de una cultura deportiva.

- $(54,33 \%)$ La integración de la comunidad.

- $(54 \%)$ ofrecer a la comunidad un espacio para la recreación y el esparcimiento.

- $(41,66 \%)$ La creación de experiencias de autogestión.

- $(37,33 \%)$ La consolidación de las organizaciones sociales sin fines de lucro.

- $(33,99 \%)$ El uso del dinero y de otros recursos sólo como medio de sostenimiento de la actividad.

En la actitud de quienes gerencian la actividad física y deportiva en las comunidades con los criterios de autosustentabilidad, así como en aquellos atletas y colaboradores que se involucran en la experiencia encontramos un alto valor altruista y esto es referente de una categoría tal vez olvidada o ignorada en estos tiempos, y es la espiritualidad humanista.

La espiritualidad humanista la configura toda esa serie de acciones, actitudes y voluntad de hacer las cosas placenteramente sin esperar nada a cambio y sin convertir la creatividad y la producción humana en objeto, fetiche o mercancía. Esto lo afirmamos muy en la óptica de (Ávila-Fuenmayor, 2011).

En ese mismo sentido, al consultar a los entrevistados y hacer la pregunta abierta: ¿Qué acciones cree usted son necesarias para darle sustentabilidad a las actividades de masificación deportiva donde usted y su 
equipo participan?. Mencione 5 indicadores o acciones; encontramos los siguientes resultados:

- $(64,33 \%)$ Acompañamiento técnico y de capacitación integral.

- $(61,66 \%)$ Mayor promoción, proyección, publicidad y cobertura en los medios de comunicación y redes sociales.

- $(60 \%)$ Mayor apoyo por parte del gobierno.

- $(51,99 \%)$ Mayor apoyo de las empresas privadas.

- $(41 \%)$ Tomar en cuenta a las instituciones educativas

- $(34,66 \%)$ Solicitar colaboración a las pequeñas empresas y establecimientos comerciales que funcionan en los barrios)

- $(34,33 \%)$ Acudir a las emisoras comunitarias para que realicen transmisiones en vivo de las competencias)

- $(29,99 \%)$ Mayor presencia de los organismos de seguridad del Estado.

Estas referencias reales y concretas son las que nos motivan no sólo a categorizarlas académicamente; sino a realizar una opción al momento de producirse el proceso de elección del tema tratado en este artículo, y al mismo tiempo en el hecho de arriesgarnos a hacer una propuesta de acción que acompañe experiencias de gerencia a la masificación deportiva planificada por las organizaciones sociales y comunitarias con criterio de autogestión y permanencia en el tiempo.

5. Propuesta de un modelo de gerencia a la masificación deportiva planificada por las organizaciones sociales y comunitarias desde la lógica del acompañamiento.

En base a la observación, comprensión y constatación de los procesos de planificación, preparación, promoción y ejecución de estas referencias de gerencia para la masificación deportiva aplicadas por organizaciones sociales 
en comunidades del estado Yaracuy, se hace la propuesta de acompañamiento con la clara y decidida posibilidad de ofrecerla sistemáticamente a promotores y dirigentes sociales involucrados en la actividad física y deportiva y con la opción de vincular tal propuesta bajo la figura de programa de extensión universitaria dirigido a las comunidades y sus actores-creadores de un modelo cultural deportivo y en parámetros de las organizaciones y empresas emergentes, según (Echeverría, 2000) y (Acosta, 2012).

Esta propuesta se construye a partir del diseño curricular de un plan flexible, abierto y andragógico en los parámetros de aprender haciendo y la sistematización y reconocimiento de experiencias.

En tal sentido, dicho plan se ajusta a un diseño curricular, según lo indica (Frías 2009), que toma en cuenta, el perfil del participante, la misión y visión de la institución que certifica el programa y el entorno o campo de acción.

Otra característica de la propuesta es la transversalidad, de la que afirman (Villanueva y Rebolledo, 2011), como aspecto estratégico en los procesos de aprendizaje, sistematización y reconocimiento de experiencias creativas, análisis e interpretación de actividades y situaciones, construcción de saberes y elaboración de propuestas pertinentes para la incidencia de experiencias formativas en la organización social, expuestas también en los lineamientos de (Martínez, 2012), (Molina 2007) y (Palos, 2010), en cuanto a las características de un diseño curricular abierto a la comunidad.

\section{Conclusiones}

El sentido estratégico de esta propuesta de acompañamiento a referencias-experienciales de gerencia en masificación deportiva desde la organización social (no estatal, no empresarial) se centra en las siguientes conclusiones: 
La masificación deportiva planificada por organizaciones sociales de las comunidades mencionadas como referentes son experiencias de autosustentabilidad, participación, intercambio, trabajo voluntario y constancia por mantener en el tiempo y en el espacio la actividad física.

Aunque desde el punto de vista de la gerencia, tales experiencias de masificación deportiva se han sostenido desde el momento de su inicio; es necesario, para consolidarlas, crear mecanismos de acompañamiento integral, en lo formativo y en la capacitación donde participen los agentes involucrados en dichos programas de masificación deportiva.

La gerencia deportiva para la masificación deportiva planificada y ejecutada por las organizaciones sociales constituye un modelo de referencia para impulsar ese tipo de experiencias en otros espacios y comunidades; puesto que sería incalculable el aporte no sólo en el desarrollo de la actividad física y deportiva; sino al mismo tiempo en proceso simultáneo contribuye una herramienta preventiva integral y de transformación social.

De éstas conclusiones se desprenden las tareas que a continuación se mencionan:

- Generar un acompañamiento técnico y metodológico a los gerentes deportivos de las organizaciones sociales y comunitarias en los procesos que fluyen a partir de la planificación y ejecución de referentes experienciales de actividad física y deportiva.

- Contribuir desde la ejecución de una propuesta formativa, con la permanencia en el tiempo y su expansión en otros espacios y territorios, e experiencias de eventos deportivos planificados y ejecutados por organizaciones deportivas.

- Sistematizar insumos, datos, estadísticas y procesos que sirvan de base informativa para otras investigaciones. 
- Posicionar en los medios y en las redes sociales estas experiencias para estimular el apoyo estatal y del sector privado, respetando el carácter autogestionario y la iniciativa genuina de las organizaciones sociales.

- Desarrollar nuevos modelos y esquemas de gerencia deportiva estatal y empresarial que incorpore y apoye las referencias gerenciales de las organizaciones deportivas sociales.

- Incentivar el apoyo financiero, material y logístico de parte del Estado, empresariado y comunidades organizadas.

\section{Referencias}

Acosta, R. (2012). Metódica de la Planificación Comunal. Caracas: Fondo Editorial Ipasme.

Ávila-Fuenmayor, F. (2006). El concepto de poder en Michel Foucault. Revista Telos, Vol. 8 No 2, pp. 215-234. Maracaibo: Universidad Dr. Rafael Belloso Chacín.

Briceño, G. (2000). Liderazgo Integral para el Progreso. Caracas: Fondo Editorial Ipasme.

Bustamante, S. (2008). Contribución teórico-metodológica al estudio de la capacidad innovativa en organizaciones de educación superior. Trabajo de ascenso para optar a la categoría de Profesor Titular. UPEL-IMPM. Caracas: Fondo Editorial de la Universidad Pedagógica Experimental Libertador.

Cagigal, J. (1981). ¡ Oh deporte! Anatomía de un gigante. Valladolid: Editorial Miñón.

Borrel, C., y Cañadas, L. (2010). El deporte como espectáculo. Buenos Aires: Ediciones Granica S.A. 
Echeverría, R. (2000). La empresa emergente, la confianza y los desafíos de la transformación. Buenos Aires: Ediciones Granica S.A.

Frías, M. (2009). Diseño y desarrollo curricular. Madrid: Ediciones Narcea. Hsu, L. (2000). From physical education to sports education? A possible tendency. Los Angeles: Bulletin of Physical Education, UCLA.

Martínez, N. (2012). La educación para la paz como eje transversal en el nivel medio superior. Tesis Doctoral UNAM. México: Libros UNAM.

Molina, D. (2007). Ejes transversales en el currículo universitario: experiencia en la carrera de derecho. Barinas: Fondo Editorial de la Universidad Nacional Experimental de los Llanos Occidentales Ezequiel Zamora.

Olivera, J. (2006). Hacia una nueva comprensión del deporte. Factores endógenos y exógenos. Apuntes: Educación Física y Deportes (86), 3-6. México: Libros UNAM.

Palos, J. (2010). Educar para el futuro: Temas transversales del currículum. Bilbao: Desclée de Brouwer.

Paredes, J. (2002). El deporte como juego: un análisis cultural. Tesis doctoral. Universidad de Alicante. Alicante: Publicaciones de la Universidad de Alicante.

Pfeffer, J. (1993). El poder en las organizaciones: Política e influencia en una empresa. Madrid: Editorial Serie McGraw-Hill de Management.

Rodríguez, J. (2002). Gerencia Deportiva Municipal. Caracas: Instituto Municipal de Publicaciones.

Senge, M. (1990). La Quinta Disciplina. El arte y la práctica de la organización abierta al aprendizaje. México: Editorial Granica. 


\section{Lorenza Antonia Reyes de Duran}

e-mail: lorenzareyes1@yahoo.com

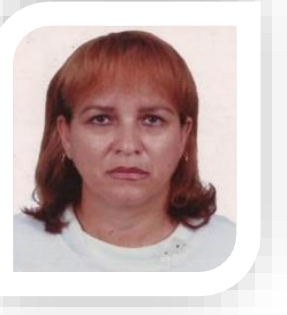

Nacida en San Felipe Yaracuy, estudia el postdoctorado en Estudios Libres en la universidad Fermín Toro en Cabudare estado Lara (2016). Doctorado en Innovaciones Educativas Universidad Nacional Experimental Politécnica de la Fuerza Armada, núcleo Yaracuy (2013). Magister Scientiarum en Gerencia Y Liderazgo en Educación en la universidad Fermín Toro núcleo San Felipe (2007). Profesora de Educación Universidad Pedagógica Experimental de Barquisimeto (1985. Maestra de Educación Primaria Mención Deportes U.E Juan José de Maya (1980). Actualmente se desempeña como docente en la catedra de Gimnasia y Atletismo en la Universidad Nacional Experimental del Yaracuy. Secretaria de Eventos de la Federación Venezolana de Gimnasia desde (2005) hasta la presente fecha Representante del Colegio de Profesores Yaracuy en la Junta Calificadora Zonal desde (1997 al 2001). Fundadora y Presidenta de la Asociación de Gimnasia del Estado Yaracuy (1985 al 1999). Integrante de la Comisión Técnica Deportiva en la Junta Directiva del Colegio de Profesores de Venezuela Seccional Yaracuy. Coordinadora deportiva del Departamento de Educación Física de la Zona Educativa, desde (1986 al 1996).

El contenido de este manuscrito se difunde bajo una Licencia de Creative Commons ReconocimientoNoComercial-Compartirlgual 4.0 Internacional 